\title{
Understanding Attributes that Influence Physician and Caregiver Decisions About Neurotechnology for Pediatric Drug-Resistant Epilepsy: A Formative Qualitative Study to Support the Development of a Discrete Choice Experiment
}

\author{
Glory Apantaku ${ }^{1}$ Magda Aguiar ${ }^{1} \cdot$ K. Julia Kaal ${ }^{1} \cdot$ Patrick J. McDonald $^{2,4} \cdot$ Mary B. Connolly $^{3} \cdot$ Viorica Hrincu $^{2} \cdot$ \\ Judy Illes ${ }^{2} \cdot$ Mark Harrison ${ }^{1,5}$ (1)
}

Accepted: 22 July 2021 / Published online: 25 August 2021

(c) The Author(s) 2021

\begin{abstract}
Objective This study reports formative qualitative research used to analyze decision making regarding neurotechnological interventions for pediatric drug-resistant epilepsy from the perspective of physicians and caregivers and the derivation of attributes for a discrete choice experiment.

Methods Purposive and convenience sampling was used to recruit physicians and caregivers. Physician focus group sessions were held at key national conferences in the USA and Canada. Caregivers were approached through clinics with established epilepsy surgery programs in the USA and Canada. Thematic analysis was used to identify critical features of decisions about treatment outcomes, procedural trade-offs, values, and concerns surrounding conventional and novel pediatric drug-resistant epilepsy interventions among physicians and caregivers.

Results The results highlight the presence of central attributes that are considered by both groups in decision making, such as "chances of seizure freedom", "risk", "availability of evidence", and "cost to families", as well as attributes that reflect important differences between groups. Physicians were focused on the specifics of treatment options, while caregivers thought more holistically, considering the overall well-being of their children.

Discussion The findings shaped the development of a discrete choice experiment to understand the likely uptake of different neurotechnologies. We identified differences in decision making and thus designed two discrete choice experiments to elicit preferences for pediatric drug-resistant epilepsy treatments, one aimed at clinicians and one at caregivers. The variation we observed highlights the value of seeking to understand the influences at the point of clinical decision making and incorporating this information into care.
\end{abstract}

\section{Introduction}

Preference-sensitive care describes circumstances where multiple, equally valid, treatment options exist [1]. In these circumstances, the best option will depend on patients, together with their clinicians, choosing the treatment that aligns best with their preferences. These preferences can be influenced by outcomes (potential benefits and harms), as well as processes (e.g., the way treatment is delivered), and

Glory Apantaku and Magda Aguiar Joint first authors.

Judy Illes

jilles@mail.ubc.ca

$\triangle$ Mark Harrison

mark.harrison@ubc.ca

Extended author information available on the last page of the article the context (i.e., structures) in which treatment is delivered (e.g., the healthcare team) [2]. When faced with preferencesensitive care, there is an expectation that patients, or in the case of children, their caregivers should be involved in shared decision making (SDM) [3]. The latter should occur in consultation with the children depending on their age and capacity to be involved in that process. Shared decision making aims to empower patients and families through information about treatments to make choices that reflect their preferences and values [3].

Although SDM has become the gold standard in clinical practice, there is no consensus on its definition [4]. Charles et al. describe the goals of SDM as "patients and healthcare professionals sharing the process of decisionmaking and ownership of the decisions made" $[5,6]$. There has been extensive work to develop decision aids to help 


\section{Key Points for Decision Makers}

Understanding the factors that are most important to people when making decisions about healthcare and how these differ between patients and physicians is an important prerequisite for shared decision making and the study of patient and physician preferences.

This study documents the process of understanding the treatment-related priorities of physicians and caregivers in the context of neurotechnological interventions for pediatric drug-resistant epilepsy using focus groups.

Our findings add to the growing literature that physicians prioritize different aspects of healthcare interventions to those of patients, and in this study, their caregivers.

patients better understand treatment options, clarify their personal values and preferences, and communicate these to clinicians $[3,7]$. The careful consideration and reflection of patient perspectives and values in the treatment process is key to successful SDM [8]. Understanding what matters to patients - the preference diagnosis [9] — can be as important as the medical diagnosis [10]. A key limitation of preference diagnosis is that healthcare professionals often do not understand what is most important to patients or their caregivers, whilst simultaneously perceiving themselves to be good at diagnosing patient preferences [11]. One recommended step toward improving preference diagnosis is to first understand the preferences of patients for treatment and then to document how they differ from preferences of clinicians [11].

Studies of patient and physician decision making have proliferated in recent years and choice experiments, especially discrete choice experiments (DCEs), have become popular [12]. Discrete choice experiments elicit the preferences of participants based on their choices between hypothetical treatments and services, described by key features known as attributes [13]. Growing evidence from DCEs suggests that the decision process of physicians/clinicians is often different from those of patients or other stakeholders involved in treatment decisions [14]. However, few studies directly compared preferences of multiple groups of stakeholders to explore how and why these preferences differ. Developing a DCE offers an important opportunity to understand differences in patient and physician preferences. Discrete choice experiments should include attributes that are most important to the target populations, therefore best practice recommends qualitative research in all potential respondent groups to understand the factors that are considered in decision making [15]. The qualitative work used to develop these DCEs is rarely formally reported; potentially valuable information about the decision-making processes is therefore lost [16]. A recent guideline recommends best practice for reporting the qualitative methods used in the development of attributes in DCEs [16].

This study reports qualitative research on decision making by physicians and caregivers to derive attributes for a DCE about neurotechnology treatment options for pediatric drug-resistant epilepsy (DRE). We use data collected from focus groups, which sought to explore the values and priorities that influence the decision-making process of physician and caregivers; the broader ethical context of these decisions has been documented elsewhere [17, 18], and, in this study, we focus on the features of treatments that drive decision making. We hypothesized that pediatric neurosurgeons, pediatric neurologists, and caregivers acting on behalf of minors follow different decision pathways and might even pursue different outcomes in approaching the same decision. We report the qualitative approach taken to identify attributes using the guidelines for best practice in developing DCEs [2, 15, 16], and qualitative findings on how conceptually different these groups perceive the same choice and how this influenced our methodological decisions.

\section{Methods}

\subsection{Setting}

Up to $30 \%$ of children with epilepsy do not respond to antiseizure medications, a condition known as DRE. In some studies, up to $70 \%$ of carefully selected patients with DRE become seizure free after epilepsy surgery [19]. Technological advances have resulted in less invasive therapies to treat DRE. Some replace more invasive surgical treatments, while others provide options for surgical treatment for conditions initially thought to be not amenable to surgery [20]. Decisions about these novel neurotechnologies by healthcare providers and parents are often made in the context of incomplete evidence regarding the efficacy, safety, and long-term side effects as few innovative neurosurgical interventions are put through the rigors of a randomized controlled trial before being adopted into practice [21].

\subsection{Qualitative Approach}

We engaged clinicians and caregivers using qualitative focus group methodology to identify critical features for decisions about treatment outcomes and procedural trade-offs, values, and concerns for decision making surrounding conventional and novel interventions for DRE using neurotechnology. Focus groups were chosen because they encourage reflection as participants discuss shared experiences and give voice to their nuanced thought processes [22]. 
Focus group discussions were audio recorded, transcribed professionally, and then coded using an inductive approach to thematic analysis. This approach does not try to fit data into a pre-existing theoretical framework, but rather allows themes to emerge from the data [23]. The findings were applied to generate key attributes around choice and decision making to be used in a DCE to determine the relative preference for each attribute such as risks, benefits, compliance, and reversibility. We followed recent recommendations for reporting formative qualitative research to support the development of quantitative preference survey instruments [16].

\subsection{Theoretical Framework}

Discrete choice experiments are underpinned by random utility theory/Lancaster's theory of demand [24, 25]. The theory provides a framework for preference elicitation methodologies by distilling decision making into its component parts, i.e., decision making about goods/services can be decomposed into combinations of their attributes and levels, each with different values [25]. Guidelines on the development of DCEs recommend that relevant attributes and levels should be identified by qualitative research [13].

\subsection{Researcher Characteristics and Reflexivity}

This study was conducted by an interdisciplinary team of health economists, ethicists, clinicians, and health service researchers. Two members of the team have experience with DCEs and the qualitative methodology used in their development (MH, MA), and clinicians in the team (PM, MM) routinely face the decision problem in DRE that this study addresses. Focus group sessions were led by the principal investigator [PI] (JI), an ethicist or co-PI (PJM), a neurosurgeon, one local collaborator, and supported by a senior researcher or graduate student. Data analysis was conducted by three researchers (MA, KJK, and GA) who brought different expertise and experience in qualitative methodology and development of DCEs to the project. MA is a health economist and trained pharmacist, GA is a researcher, and $\mathrm{KJK}$ is a psychologist and health service researcher.

\subsection{Sampling Strategy and Process}

Pediatric neurologists and neurosurgeons were recruited purposively for their expertise to one of four focus groups of six to eight participants held at key national conferences in the USA and Canada. We also posted the opportunity to participate in the groups at the meetings as a method of convenience sampling. Caregivers of children were recruited purposively for their experience in caring for children with
DRE. In Canada, caregivers were recruited at the two largest pediatric epilepsy surgery programs in the country (BC Children's Hospital, Vancouver; The Hospital for Sick Children [SickKids], Toronto). Both are public institutions receiving referrals and caring for patients from across the country. In the USA, caregivers were recruited from the Epilepsy Clinics at UCSF Benioff Children's Hospital (San Francisco) and Monroe Carell Jr. Children's Hospital at Vanderbilt (Nashville), two high-volume epilepsy surgery centers with a history of early adoption of novel surgical interventions for DRE. Caregivers were recruited from the population of children who had a surgical intervention for DRE, regardless of the type of intervention they received. Focus groups were held onsite at these hospitals for their convenience.

\subsection{Sampling Adequacy}

Physicians were recruited by e-mail from the network of neurologists and neurosurgeons specializing in pediatric epilepsy in Canada and USA and invited to focus groups taking place at national or international specialty conferences suited to gathering the participants in each of the two countries. The groups took place at a pre-set time and date. Posted announcements about the groups at the conferences provided an opportunity for convenience sampling. Clinicians who voluntarily disclosed a financial relationship with a neurotechnology company that manufactures a product used for epilepsy surgery were excluded. Caregivers were recruited from the large epilepsy centers to maximize the diversity of perspectives and opinions. We sought to be comprehensive in data collection, with semi-structured focus group sessions designed to be fluid and responsive to participants, ensuring they explored the full range of potential factors influencing their decision making. At least one of the researchers present at each focus group session had experience in developing DCEs and was there to facilitate careful probing and inquiry on subjects that arose around decision making. The probing within focus groups continued until the researchers attending the focus groups felt they had gained a comprehensive understanding of participants' perspectives. At the analysis stage, coding continued until no new themes on the drivers of decision making were identified.

\subsection{Ethics Review}

Participants reviewed the consent form and study information material in advance of the focus group. Ethics approval for the study was granted by the University of British Columbia Behavioral Research Ethics Board (H18-02783), the SickKids Research Ethics Board (1000063826), and the Vanderbilt Institutional Review Board (190374). 


\subsection{Data Collection Methods and Sources}

A detailed account of the sampling strategy is provided elsewhere $[17,18]$. Focus group guides were developed to help gain a detailed insight into the context of making decisions in pediatric DRE. The guides were informed by a combination of a literature review of choice experiments, previous choice experiments, and studies of decision making in pediatric DRE [26-28], and input from clinical experts and research team members. The focus group guide is available in the Electronic Supplementary Material. All focus groups were 60 min long and led by the PI (JI) or co-PI (PJM), one local collaborator, and supported by a senior researcher or graduate student.

During the focus group session, clinicians explored their knowledge of neurotechnological interventions, their role in decision making, and the perception of trade-offs that exist as they explore treatment options. The caregiver focus group sessions explored similar topics as the clinician focus groups with the addition of broader considerations that affect their decision making.

This study used the same input data from focus groups as other analyses [17, 18], but applied a different qualitative methodology and framework to support the development of the DCE. The primary objective of the qualitative research was to support the development of the DCE but the richness of discussion allowed other contextual factors, which would not be considered attributes, to be explored by other qualitative studies $[17,18]$.

\subsection{Data Processing and Data Analysis}

An inductive approach was used to identify themes. Researchers GA, MA, and KJK familiarized themselves with the data by reading the transcripts multiple times. They each independently coded a subset of transcripts and identified preliminary themes and patterns [23]. GA and KJK developed a coding framework. The analysis was conducted iteratively by the researchers who met routinely to conduct detailed reviews during which additional themes emerged. Disagreements were adjudicated by MA. NVIVO Version 12 was used to manage the data. All authors were involved in the review and interpretation of the data.

In developing attributes, the core question was: "does this drive decision making?" The researchers (GA, MA, and KJK) developed a list of potential attributes from themes identified by interpreting participants' description of factors that directly affected decisions to recommend or uptake treatments for DRE. This list was presented to the investigators and collaborators on the study, which included neurosurgeons and neurologists, to narrow down the final list of attributes for inclusion in the DCE. Selecting attributes is a balance between including the most important features, without providing too many attributes, which increases the complexity of the tasks for the respondents, and can result in attribute non-attendance, inconsistent responses across choice tasks, and increased dropout rates [29]. The experts and researchers screened all generated attributes and levels using multiple criteria such as decision context and plausibility. As a decision to choose no intervention was plausible, a no intervention opt-out was carefully defined.

\subsection{Techniques to Enhance Trustworthiness}

Results were presented to the full investigator team, including neurosurgeons, neurologists, ethicists, and DCE experts from the USA and Canada, who provided guidance on the most salient drivers of decision making. Attributes and levels were developed and validated with the panel of experts, the survey was then piloted with a group of neurosurgeons and caregivers who provided valuable feedback on wording and the experience of completing the survey.

\section{Results}

\subsection{Sample}

Thirty-three physicians and 22 caregivers participated in four physician and three caregiver focus groups, respectively (Table 1). The children of the 22 caregivers were $55 \%$ male, and included infants, children, preadolescents, and adolescents equally.

\subsection{Evidence}

Focus group analysis identified 12 core themes influencing physician decision making around neurotechnological interventions for DRE in pediatric patients and eight for caregivers (Table 2). The final attributes included in both DCEs, reflecting the most significant choice drivers, differed slightly between groups in number and wording. Their derivation is now described.

\subsection{Attributes Included in Both DCEs}

A number of attributes were common to both DCEs, reflecting the themes identified from both stakeholder focus groups; however, the attributes and levels were worded to fit the intended audience in consultation with physicians and caregivers, respectively. Table 3 includes quotes expressing physician and caregiver perspectives on these attributes. 


\subsubsection{Chances of Seizure Freedom}

Physicians were concerned with the efficacy of the intervention and the chances that their patients would achieve a clinically significant reduction in seizure frequency. The limited evidence on relatively new neurotechnologies was cause for concern, as their goal was to see their patients live a more seizure-free life.

Caregivers reported significant concerns about the quality of life of their children and how it could be improved by intervention. The definition of quality of life in their decision making was driven by whether their child(ren) could actually be free of debilitating seizures and able to live a normal life with a good social network.

\subsubsection{Risk (Major and Minor Risk)}

Physicians discussed their concerns through a number of themes: risk, safety, mortality, and invasiveness. Physicians described considering new technologies only if they were safe, proven to have very low mortality rates, and would provide some level of health improvement. They also noted that they had to weigh and communicate to caregivers the risk of an intervention based on the invasiveness of the surgery. Additionally, they were aware of both the risk associated with the intervention and the risk of not intervening, namely, ongoing uncontrolled seizures, which they considered to be significant. These themes were considered to be sufficiently captured under the 'risk' and 'chances of seizure freedom' attributes. The risk theme was split into two attributes describing minor and major risks, to sufficiently capture the complexity of the risk considerations, to provide clarity on the specifics of the risk factors, and for a better understanding of the trade-offs people make between benefits and risks of different severity and the likelihood of occurrence.

For caregivers, balancing risk and benefit was also a salient factor they considered, they recognized the risk of doing nothing and described making choices based on whether the intervention was more or less risky compared to the natural history of uncontrolled epilepsy. The themes of risk described by caregivers were also split into two attributes to replicate the question in the physician DCE, to provide consistency, and to allow for a more direct comparison.

\subsubsection{Availability of Evidence}

The 'source of information' theme captured physician concerns about the source, quality, and trustworthiness of evidence supporting possible interventions. With novel surgical
Table 1 Demographic data

\begin{tabular}{|c|c|c|c|c|}
\hline \multirow[t]{2}{*}{ Characteristics } & \multicolumn{2}{|c|}{$\begin{array}{l}\text { Physicians } \\
(n=33)\end{array}$} & \multicolumn{2}{|c|}{$\begin{array}{l}\text { Caregivers } \\
(n=22)\end{array}$} \\
\hline & $n$ & $\%$ & $n$ & $\%$ \\
\hline \multicolumn{5}{|l|}{ Sex } \\
\hline Female & 9 & 27 & 18 & 82 \\
\hline Male & 18 & 55 & 4 & 18 \\
\hline Missing & 4 & 12 & - & - \\
\hline \multicolumn{5}{|l|}{ Age group, years } \\
\hline $31-40$ & 5 & 15 & 1 & 5 \\
\hline $41-50$ & 12 & 36 & 5 & 23 \\
\hline $51-60$ & 5 & 15 & 8 & 36 \\
\hline 61 and above & 5 & 15 & 8 & 36 \\
\hline Missing & 6 & 18 & - & - \\
\hline \multicolumn{5}{|l|}{ Race/ethnicity } \\
\hline White & 19 & 58 & 16 & 73 \\
\hline Asian & 6 & 18 & 2 & 9 \\
\hline Black & 2 & 6 & - & - \\
\hline Hispanic & 1 & 3 & 1 & 5 \\
\hline Other & 1 & 3 & - & - \\
\hline Missing & 4 & 12 & 3 & 14 \\
\hline \multicolumn{5}{|l|}{ Location } \\
\hline USA & 12 & 36 & 8 & 36 \\
\hline Canada & 17 & 52 & 12 & 55 \\
\hline Missing & 4 & 12 & 2 & 9 \\
\hline \multicolumn{5}{|l|}{ Type of practice } \\
\hline Private & 1 & 3 & - & - \\
\hline Public & 23 & 70 & - & - \\
\hline Public/private & 4 & 12 & - & - \\
\hline Missing & 5 & 15 & & \\
\hline \multicolumn{5}{|l|}{ Years in practice } \\
\hline$<1-5$ & 7 & 21 & - & - \\
\hline $5-10$ & 6 & 18 & - & - \\
\hline $10-15$ & 3 & 9 & - & - \\
\hline $15-20$ & 2 & 6 & - & - \\
\hline 21 and above & 6 & 18 & - & - \\
\hline Missing & 9 & 27 & - & - \\
\hline \multicolumn{5}{|l|}{ Education } \\
\hline High school degree or equivalent & - & - & 4 & 18 \\
\hline Some college but no degree & - & - & 4 & 18 \\
\hline Bachelor's degree & - & - & 8 & 36 \\
\hline Graduate degree & - & - & 4 & 18 \\
\hline Missing & - & - & 2 & 9 \\
\hline \multicolumn{5}{|l|}{ Total household income (US\$) } \\
\hline Less than 50,000 & - & - & 1 & 5 \\
\hline $50,000-100,000$ & - & - & 6 & 27 \\
\hline 100,000 or more & - & - & 12 & 54 \\
\hline Prefer not to say & - & - & 3 & 14 \\
\hline
\end{tabular}


Table 2 Physician and caregiver themes and attributes

\begin{tabular}{|c|c|c|c|}
\hline \multicolumn{2}{|l|}{ Physician DCE } & \multicolumn{2}{|l|}{ Caregiver DCE } \\
\hline Themes & Attributes & Attributes & Themes \\
\hline 1. Efficacy & 1. Chance of seizure freedom & 1. Chance of seizure freedom & 1. Quality of life \\
\hline 2. Risks & 2. Minor risks & 3. Major risks & 2. Risk \\
\hline \multicolumn{4}{|l|}{ 3. Safety } \\
\hline \multicolumn{4}{|l|}{ 4. Mortality } \\
\hline \multicolumn{4}{|l|}{ 5. Invasiveness } \\
\hline 6. Source of information & 4. Evidence & 4. Science supporting the intervention & $\begin{array}{l}\text { 3. Evidence, experience of } \\
\text { physician }\end{array}$ \\
\hline $\begin{array}{l}\text { 7. Cost and resource use } \\
8 \text {. Burden to family }\end{array}$ & 5. Financial burden on family & $\begin{array}{l}\text { 5. Burden of out-of-pocket payments on } \\
\text { your finances }\end{array}$ & 4. Cost \\
\hline $\begin{array}{l}\text { 9. Availability at institu- } \\
\text { tion level }\end{array}$ & 6. Availability at institution level & 6. Access to multidisciplinary care team & $\begin{array}{l}\text { 5. Access to multidiscipli- } \\
\text { nary care team }\end{array}$ \\
\hline \multicolumn{4}{|l|}{$\begin{array}{l}\text { 10. Care team character- } \\
\text { istics }\end{array}$} \\
\hline $\begin{array}{l}\text { 11. Family preference or } \\
\text { request for treatment }\end{array}$ & N/A & 7. Invasiveness & 6. Invasiveness \\
\hline 12. Pressure to innovate & N/A & N/A & $\begin{array}{l}\text { 7. Involving the child, } \\
\text { patient in decision mak- } \\
\text { ing }\end{array}$ \\
\hline 13. Appropriateness & N/A & N/A & $\begin{array}{l}\text { 8. Trust in physician, } \\
\text { medical institution }\end{array}$ \\
\hline
\end{tabular}

$D C E$ discrete choice experiment, $N / A$ theme did not translate to an attribute

interventions, where rigorous studies such as clinical trials are usually limited, physicians considered anecdotal evidence useful when it came from respected colleagues with significant experience and expertise in well-regarded institutions. This theme was linked with 'evidence' and described as 'availability of evidence'.

Caregivers also considered the available evidence and were interested in knowing the types of studies and the health outcomes of patients who had received the interventions they were considering for their children. Worries about being a test case or the first to have a procedure was also raised by caregivers.

\subsubsection{Cost to Families}

This attribute described as the 'financial burden to families' in the physician DCE was based on our thematic analysis showing that physicians were aware of the possible direct financial cost families may incur in caring for children with DRE, along with the logistical burdens of transport and travel to receive specific interventions in larger centers, often far from home. These issues factored into the physician decisions about whether they would recommend certain interventions.

For caregivers, cost was also a salient concern, given the significant costs associated with these interventions and the complicated nature of insurance coverage, especially in the
US focus groups. Caregivers in the USA were especially worried about the implications of a loss of employment on access to high-quality medical insurance to care for their children.

\subsection{Attributes Included Only in Physician or Caregiver DCEs}

A small number of attributes appeared to be only key drivers of decisions for either physicians or caregivers (Table 4).

\subsubsection{Availability of Intervention at Institution Level (Physicians)}

Physicians were very aware of the features of novel neurotechnologies and how to deliver them to patients. However, they noted that the availability of the intervention within their institution would determine their ability to deliver or recommend an intervention, especially because referring patients to other centers could mean increasing the financial burden (because of time lost at work, travel, accommodation) on caregivers.

\subsubsection{Access to a Multidisciplinary Care Team (Caregivers)}

Caregivers described a need for additional support outside their epilepsy surgery teams in caring for their children with 


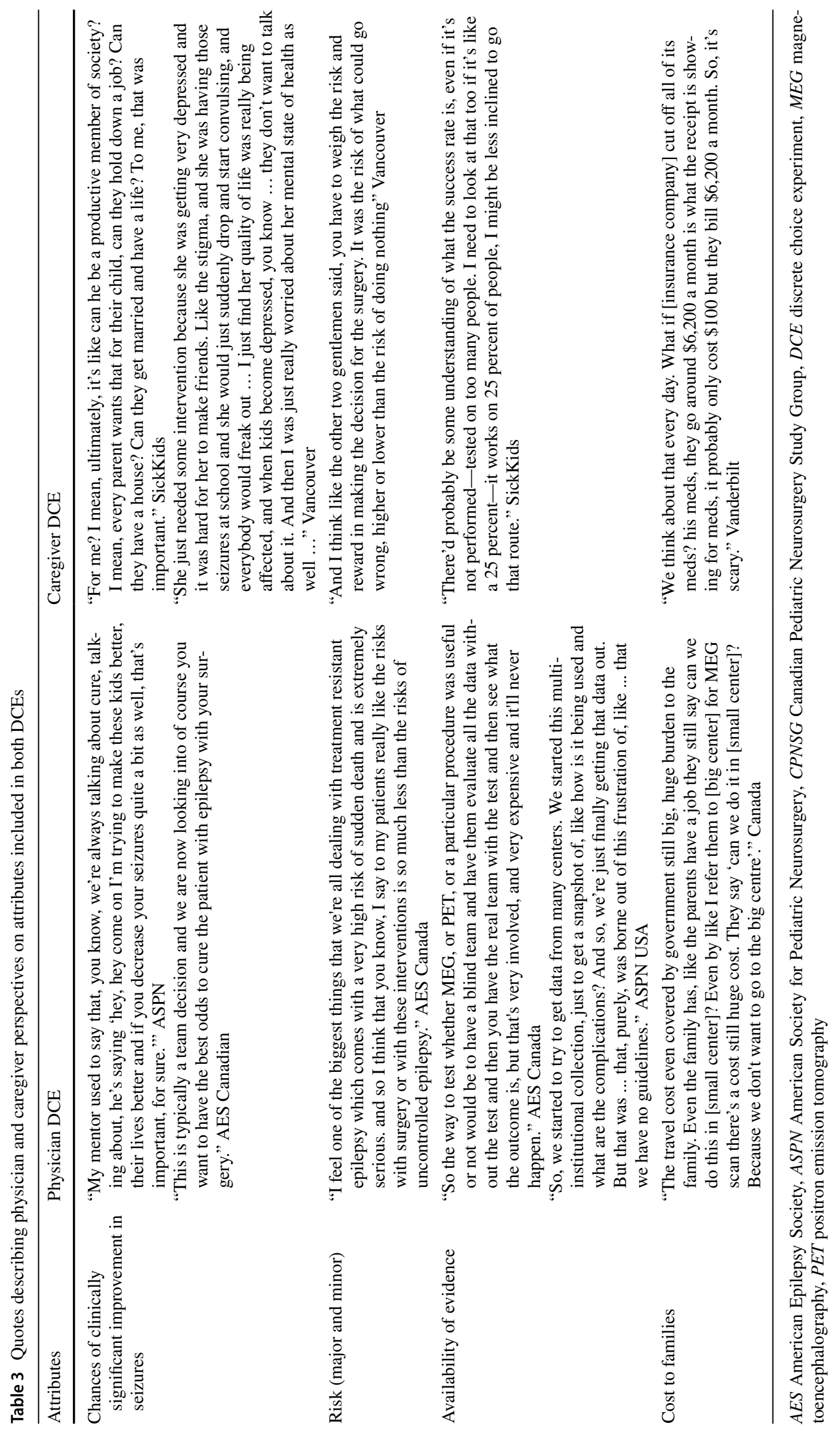


DRE, and often expressed a desire for access to other healthcare professionals, which ranged from counsellors to support their children with the psychological aspects of their disease to social workers for support with other aspects of life.

\subsubsection{Invasiveness (Caregivers)}

Caregivers frequently discussed concerns about the level of invasiveness of procedures and how novel technologies seemed less invasive and therefore favorable. Conversations from multiple focus groups suggested that preferences for interventions could be influenced by the degree of invasiveness.

\subsection{Themes that did not Translate to Attributes}

Under the 'burden to family' theme, physicians expressed concerns about travel costs for caregivers and their ability to complete the treatment process. As this theme appeared to be inextricably linked with cost, this theme contributed to the 'financial burden on family' attribute.

'Pressure to innovate' was primarily discussed by American physicians, but not by their Canadian counterparts. This theme related closely to the 'availability of intervention at institution level' attribute. Similarly, physicians discussed 'family preferences' as a theme, describing feelings of being burdened or pressured by the desires of the patients' family

Table 4 Quotes describing physician and caregiver perspectives on attributes

\begin{tabular}{|c|c|c|}
\hline Attributes & Physician DCE & Caregiver DCE \\
\hline $\begin{array}{l}\text { Availability of } \\
\text { intervention at } \\
\text { institution level }\end{array}$ & $\begin{array}{l}\text { "We don't have any of this in [city] ... it's difficult as a surgeon in an } \\
\text { institution that does not have any of this technology, VNS aside. It's } \\
\text { very difficult to know how to recommend treatment plans to patients. } \\
\text { Now we don't get asked the question a lot but we don't have a program. } \\
\text { But, so you have a laser in [city] or you have the depth electrodes, do I } \\
\text { send the patient somewhere else, how do I choose? Because at the end } \\
\text { of the day what sometimes happens is the patient comes in and says, } \\
\text { 'Have you heard about this new technology, this interstitial laser?' And } \\
\text { I say, 'Well, yes, I've heard about it but I don't have any experience of } \\
\text { it."' CPNS Canada }\end{array}$ & \\
\hline $\begin{array}{l}\text { Access to a multi- } \\
\text { disciplinary care } \\
\text { team }\end{array}$ & & $\begin{array}{l}\text { "So just having one person with their only } \\
\text { opinion and stuff versus like a whole group } \\
\text { of people and they've all talked about it and } \\
\text { they've all shared ideas and I just feel like you } \\
\text { get a lot more reassurance from having many } \\
\text { people who are kind of on the same page. And } \\
\text { again, yeah, like even now I can still email the } \\
\text { nurse practitioner or anybody else and get an } \\
\text { answer if you have problems. It's nice know- } \\
\text { ing that you have that group there." SickKids } \\
\text { "Yeah, I felt like maybe a social worker to be } \\
\text { sort of a liaison with families and physicians } \\
\text { and other services that need to be tapped into. } \\
\text { We tried to care for a child with significant } \\
\text { special needs and the rest of your family and } \\
\text { work and then trying to make calls to agen- } \\
\text { cies and it's just is a lot to be on edge. I often } \\
\text { thought that like a social worker that can help } \\
\text { families who have other areas of their lives } \\
\text { are being impacted aside from the seizures". } \\
\text { Vanderbilt }\end{array}$ \\
\hline Invasiveness & & $\begin{array}{l}\text { "I'm more comfortable with like these devices. } \\
\text { That's the one that scalp-as long as the side } \\
\text { effects, even though it's new, there's probably } \\
\text { listed side effects. So, first thing I'd look at is } \\
\text { the side effects and how invasive it is before I } \\
\text { agree to go with that." SickKids }\end{array}$ \\
\hline
\end{tabular}

CPNSG Canadian Pediatric Neurosurgery Study Group, DCE discrete choice experiment, VNS vagus nerve stimulation 
but ultimately they did not believe that this strongly influenced their recommendations or decisions.

We also identified themes such as 'trust in physicians,' 'involving the child (patient) in decision making,' and 'appropriateness of treatment' for an individual. Whilst important, these were either prerequisites to deciding about any type of treatment ('appropriateness'; 'trust in physician'), or were inherent to the physician-patient relationship and unlikely to vary between treatments ('trust in physicians'; 'involving the child').

\subsection{Synthesis/Interpretation}

Guided by our core question "does this drive decision making?", we worked to identify themes that directly affected decision making and varied between options, and then described those key concepts in a minimum set of attributes (Table 5). Overall, physicians prioritized improving the quality of lives of the patients by reducing their seizures, but in a way that would not leave caregivers facing serious financial pressures. Caregivers were similarly focused on long-term outcomes associated with the interventions, and their thought process revolved very much around their child's quality of life post-intervention, as well as access to comprehensive care.

\section{Discussion}

In this qualitative study, we explored how clinicians and caregivers contemplate the decision between traditional epilepsy surgery and novel neurotechnologies to treat a child with DRE. We found that there are central attributes in decision making that are common to both groups, as well as attributes that reflect important differences. Physicians were focused on the specifics of treatment options, and the availability and quality of evidence supporting their efficacy. Caregivers of children with DRE were thinking more holistically, considering the overall well-being of their child. Furthermore, even where attributes can be described in a way that is common to both, there were subtle but important differences in the themes that generated these attributes. The current study also provides documentation of the process we used to develop attributes for a DCE through qualitative methods.

Our finding that physicians prioritize different aspects of healthcare interventions is consistent with findings from a review of patient and physician preferences from DCEs [14]. The implication of differences in decision making led us to design two different DCEs to elicit preferences for DRE treatments: one aimed at clinicians and one at caregivers, albeit with some common attributes. This distinction allowed us to remain true to the focus group discussions that we analyzed. When designing a DCE to survey different groups of the population, the decision to use the same survey with the same attributes and wording, or to tailor the survey to each population group is important, and methodological trade-offs must be considered. Using different versions for different groups limits the comparability of the results: it cannot be assessed if differences in the preferences (i.e., in the attribute level coefficients) between groups are due to genuine differences or to the choice sets they completed because of different attributes, levels, or framing/wording of any of these aspects [14]. However, using the same questionnaire and thereby forcing researchers to ignore fundamental differences in how groups might perceive a decision and in the attributes that matter for each group, would directly conflict with best practice to develop DCEs [13]. For this reason, we believed it was more appropriate to use attributes and language that most closely replicate the factors driving decision making identified through our qualitative work. Whilst this precludes direct comparisons of preferences for attributes of treatment, the inclusion of an opt-out choice in the DCE will allow us to make predictions of uptake of novel technologies by each respondent group based on their preferences for the set of attributes they use to make decisions. These predictions of uptake, based on the aspects of treatment that are relevant to each group, can inform indirect comparisons of the treatments that patients and caregivers might prefer with those that physicians might prefer.

Our inductive thematic analysis is consistent with the objective of deriving attributes that are reflective of the factors considered in decision making of physicians and caregivers. The analysis generated qualitative insights that supported the goal of generating attributes that represent the component parts of decision making. These components are situated within broader individual, social, and relational factors that have been described previously using a pragmatic neuroethics framework, and a qualitative content analysis approach to examine the balance between clinical and ethical contexts in which decisions are made [17, 18]. For example, the physician context is described as a balance between clinical processes that lead to physicians being comfortable offering a new treatment to patients, and ethical considerations of the pressure from institutions and external sources (e.g., patient groups and manufacturers) to provide new technologies, whereas the perspective of parents/caregivers documented balancing the perception of the features of the intervention [17, 18]. A small proportion of the reported features naturally overlap with our analysis (e.g., effectiveness and risks); however, the main focus, the description of the broader decision-making context, does not. These three separate analyses with different frameworks and analytical approaches represent a comprehensive description of decision making between caregivers and physicians. 


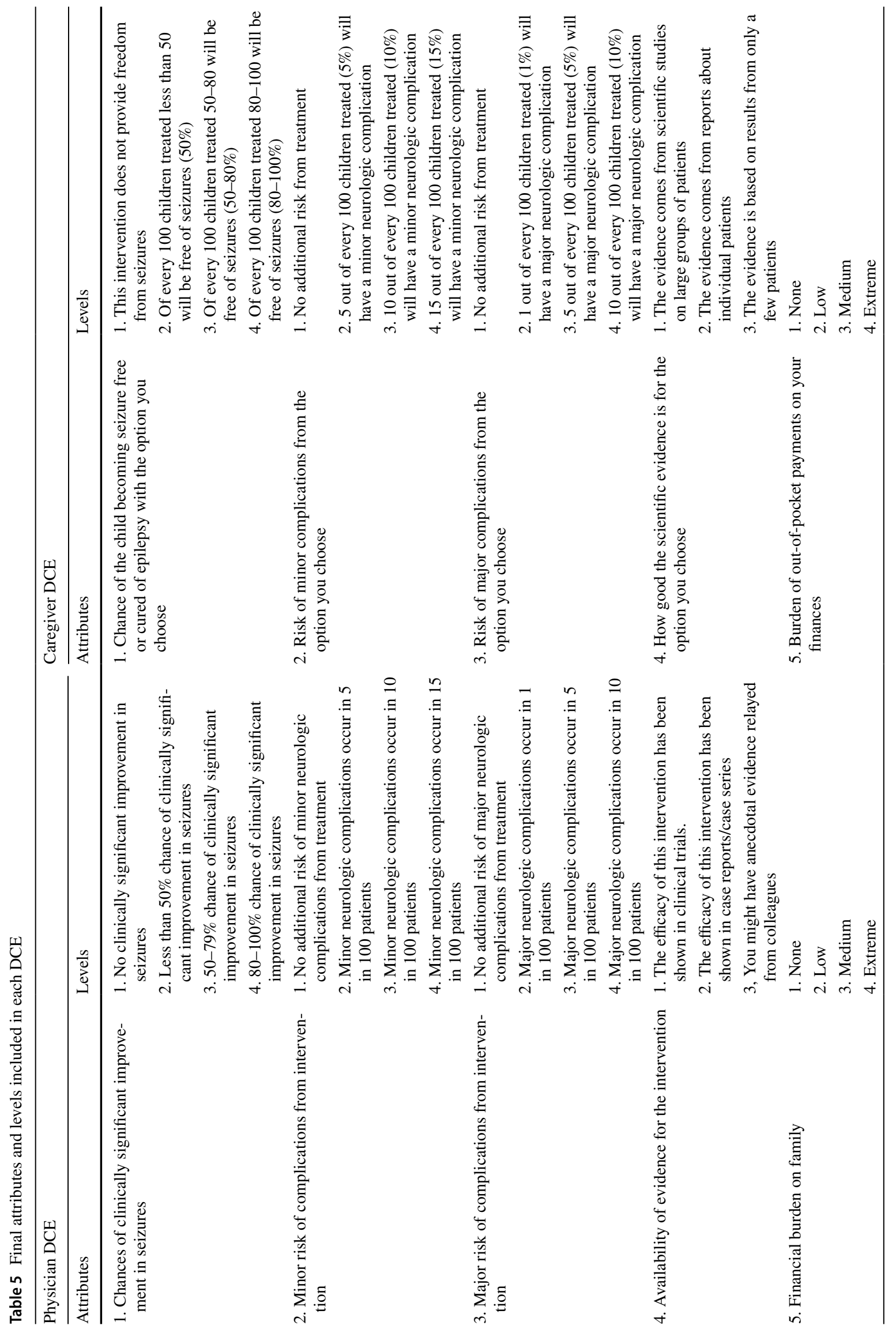




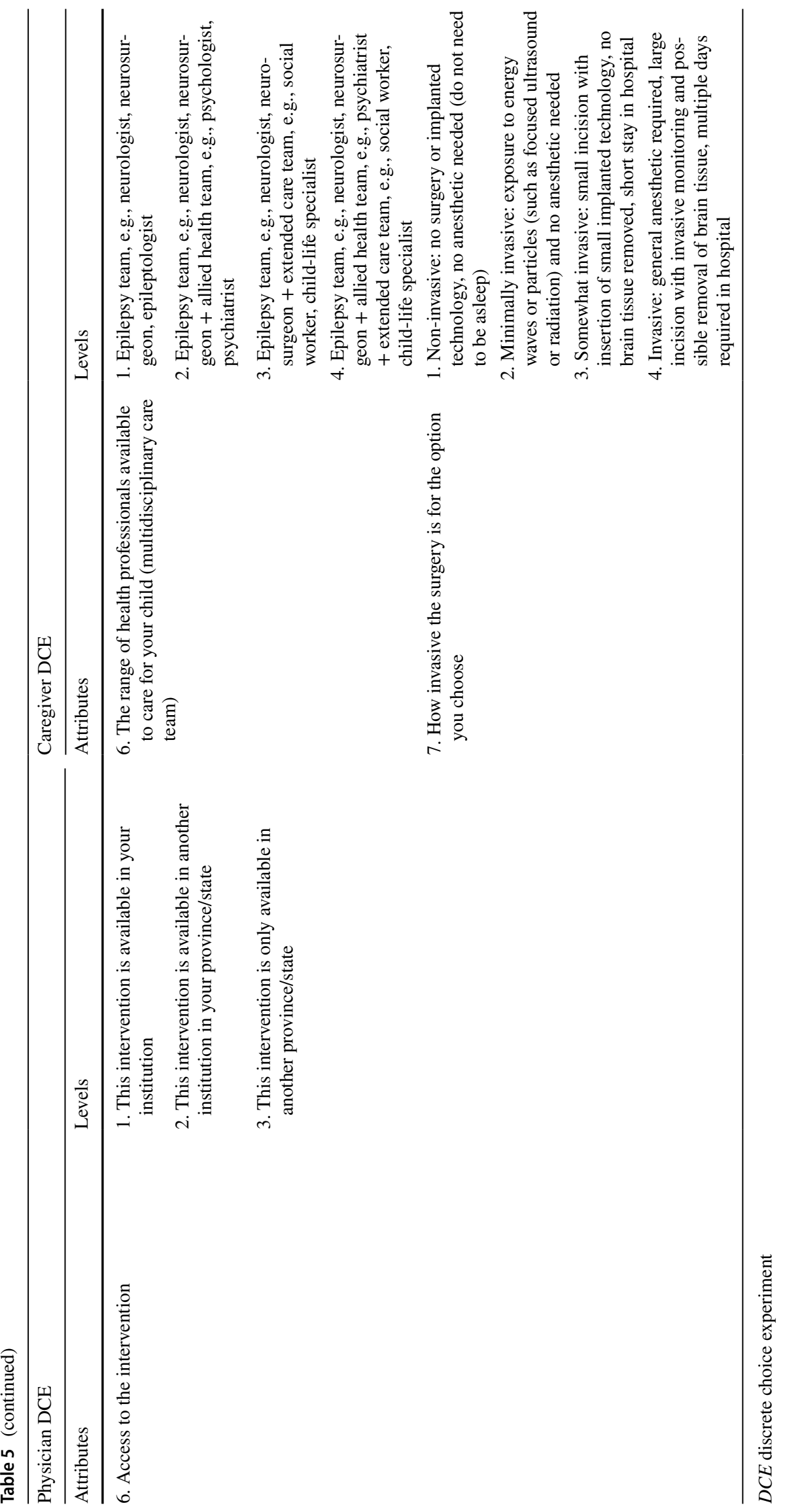


The strength of this study is the use of best practice [15] and reporting of [16] thorough and transparent approaches to qualitative work for the development of a DCE. This reporting documents the useful insights from this approach to understand differences in decision making, and supports and guides interpretation of methodological choices we made in designing the DCE. Through our thematic analysis approach, we synthesized key themes and accounted for multiple perspectives on decision making in this setting. We were also able to provide clear documentation of the sources of our attributes and the decisions made by our research team about the inclusion/exclusion of potential attributes and meaning of attributes. The varied perspectives brought by members of the multidisciplinary research team were also a strength.

Our analysis focused on the generation of attributes, and has likely missed some interesting aspects of the focus group discussion related to this specific choice context. Such insights have been published elsewhere [17, 18]. It would have been ideal to have a second round of focus groups with physicians and caregivers to review the selected list of attributes, their levels, and how best to word them. We were unable to complete this step because of time constraints, but given that the data were collected iteratively, the focus group guide evolved and probed for different drivers of decision making from participants. We have, however, piloted the resulting attributes and levels with participants from our focus groups by sending summaries of the attributes and levels we developed to participants who agreed to recontact to seek feedback to ensure they accurately reflected the focus group discussions and were understandable.

The clear finding that themes and attributes are different for caregivers and physicians has implications for SDM. The growth in evidence highlighting the value of SDM has resulted in increased attempts to foster effective communication between patients and physicians. In moving away from the paternalism in healthcare, proponents of SDM have identified the need for values clarification [8], recognizing that the assumption that patients and physicians share the same goals of treatment can be incorrect. Thus, it is essential to explore variations in the population, nuances of decision making, and the different paths physicians and patients may be willing to take to improve disease management. It is important to ensure that all the stakeholders involved in patient care acknowledge, identify, and understand the different ways a decision process unfolds for physicians and patients. This knowledge can help provide greater transparency and increase the chances of successful SDM, i.e., the patients achieve their goals and are able to engage in more effective communication with their physicians. There will very likely be areas of alignment between clinicians and their patients in objectives; however, understanding divergent patient objectives is a valuable part of SDM. The variation we observed between clinicians and caregivers underlines the importance of trying to understand the prevailing influences at the interface of patient, caregiver, and physician decision making and incorporating this information into care.

The findings presented here shaped the development and administration of two DCEs to understand the likely uptake of different neurotechnologies. This work also provides valuable information to support decision making for patients with DRE, their caregivers, and their healthcare providers in the future.

\section{Conclusions}

By exploring the most influential features of conventional and novel interventions for DRE using neurotechnology from the perspectives of physicians and caregivers, we found both a common ground and key differences. Notably, caregivers had a more holistic perspective about the overall well-being of their child. These differences can have a profound impact on developing DCEs that are designed to investigate key attributes and trade-offs people are willing to make in achieving their treatment goals.

Supplementary Information The online version contains supplementary material available at https://doi.org/10.1007/s40271-021-00544-w.

Acknowledgments The authors thank Dr. George Ibrahim, Dr. Urs Ribary, Dr. Robert Naftel, Dr. Eric Racine, Dr. Winston Chiong, and Cailin Lechner for their contributions to data collection and analysis.

\section{Declarations}

Funding This work was supported by the National Institutes of Health BRAIN Initiative on Neuroethics (RF1 \# MH1 17805 01; JI, PI; PJM, co-PI). JI is the Canada Research Chair in Neuroethics. PJM is the University of British Columbia Alcan Chair in Neurosciences at the University of British Columbia. MH received salary support through a Michael Smith Foundation for Health Research Scholar Award (16813).

Conflict of interest Glory Apantaku, Magda Aguiar, Julia Kaal, Patrick J. McDonald, Mary B. Connolly, Viorica Hrincu, Judy Illes, and Mark Harrison have no conflict of interest to disclose.

Availability of data and material The datasets generated during and/or analyzed during the current study are available from the corresponding author on reasonable request and subject to ethics approval.

Code availability Not applicable.

Author contributions Conceptualization: MA, MH, JI, PM, MC; methodology: MH, MA, KJ, GA; investigation: MA, KJ, GA, PJM, JI; writing: GA, MA, KJ, MH; funding: JI, PJM; and supervision and guarantor: MH, JI, PJM.

Ethics approval Approval for the study was granted by the University of British Columbia Behavioral Research Ethics Board (H18-02783), 
the SickKids Research Ethics Board (1000063826), and the Vanderbilt Institutional Review Board (190374).

Consent to participate Informed consent was obtained from all individual participants included in the study.

Consent for publication Not applicable.

Open Access This article is licensed under a Creative Commons Attribution-NonCommercial 4.0 International License, which permits any non-commercial use, sharing, adaptation, distribution and reproduction in any medium or format, as long as you give appropriate credit to the original author(s) and the source, provide a link to the Creative Commons licence, and indicate if changes were made. The images or other third party material in this article are included in the article's Creative Commons licence, unless indicated otherwise in a credit line to the material. If material is not included in the article's Creative Commons licence and your intended use is not permitted by statutory regulation or exceeds the permitted use, you will need to obtain permission directly from the copyright holder. To view a copy of this licence, visit http://creativecommons.org/licenses/by-nc/4.0/.

\section{References}

1. Elwyn G, Frosch D, Rollnick S. Dual equipoise shared decision making: definitions for decision and behaviour support interventions. Implement Sci. 2009;4(1):75. https://doi.org/10.1186/ 1748-5908-4-75.

2. Donabedian A. Evaluating the quality of medical care. Milbank Q. 2005;83(4):691-729.

3. Elwyn G, O'Connor A, Stacey D, Volk R, Edwards A, Coulter A, et al. Developing a quality criteria framework for patient decision aids: online international Delphi consensus process. BMJ. 2006;333(7565):417.

4. Moumjid N, Gafni A, Brémond A, Carrère M-O. Shared decision making in the medical encounter: are we all talking about the same thing? Med Decis Mak. 2007;27(5):539-46.

5. Charles C, Gafni A, Whelan T. Shared decision-making in the medical encounter: what does it mean? (or it takes at least two to tango). Soc Sci Med. 1997;44(5):681-92.

6. Charles C, Gafni A, Whelan T. Decision-making in the physicianpatient encounter: revisiting the shared treatment decision-making model. Soc Sci Med. 1999;49(5):651-61.

7. Stacey D, Légaré F, Col NF, Bennett CL, Barry MJ, Eden KB, et al. Decision aids for people facing health treatment or screening decisions. Cochrane Database Syst Rev. 2014;1:CD001431.

8. Elwyn G, Frosch D, Thomson R, Joseph-Williams N, Lloyd A, Kinnersley P, et al. Shared decision making: a model for clinical practice. J Gen Intern Med. 2012;27(10):1361-7. https://doi.org/ 10.1007/s11606-012-2077-6.

9. Godlee F. Diagnosing the patient's preference. BMJ. 2012;345:e7745.

10. Mulley AG, Trimble C, Elwyn G. Stop the silent misdiagnosis: patients' preferences matter. BMJ. 2012;345:e6572.

11. Mulley A, Trimble C, Elwyn G. Patients' preferences matter: stop the silent misdiagnosis. London: The King's Fund. https://www. kingsfund.org.uk/sites/default/files/field/field_publication_file/ patients-preferences-matter-may-2012.pdf. Accessed 27 Jul 2021.

12. Soekhai V, de Bekker-Grob EW, Ellis AR, Vass CM. Discrete choice experiments in health economics: past, present and future. Pharmacoeconomics. 2019;37(2):201-26.
13. Lancsar E, Louviere J. Conducting discrete choice experiments to inform healthcare decision making. Pharmacoeconomics. 2008;26(8):661-77. https://doi.org/10.2165/00019053-20082 6080-00004.

14. Harrison M, Milbers K, Hudson M, Bansback N. Do patients and health care providers have discordant preferences about which aspects of treatments matter most? Evidence from a systematic review of discrete choice experiments. BMJ Open. 2017;7(5):e014719.

15. Coast J, Al-Janabi H, Sutton EJ, Horrocks SA, Vosper AJ, Swancutt DR, et al. Using qualitative methods for attribute development for discrete choice experiments: issues and recommendations. Health Econ. 2012;21(6):730-41. https://doi.org/10.1002/hec. 1739.

16. Hollin IL, Craig BM, Coast J, Beusterien K, Vass C, DiSantostefano R, et al. Reporting formative qualitative research to support the development of quantitative preference study protocols and corresponding survey instruments: guidelines for authors and reviewers. Patient. 2020;13(1):121-36. https://doi.org/10.1007/ s40271-019-00401-x.

17. McDonald PJ, Hrincu V, Connolly MB, Harrison MJ, Ibrahim GM, Naftel RP, et al. Novel neurotechnological interventions for pediatric drug-resistant epilepsy: physician perspectives. J Child Neurol. 2021;36(3):222-9. https://doi.org/10.1177/0883073820 966935.

18. Hrincu V, McDonald PJ, Connolly MB, Harrison MJ, Ibrahim GM, Naftel RP, et al. Choice and trade-offs: parent decision-making for neurotechnologies for pediatric drug resistant epilepsy. $\mathbf{J}$ Child Neurol. 2021. https://doi.org/10.1177/08830738211015010.

19. Muh CR. Current and emerging surgical therapies for severe pediatric epilepsies. Semin Pediatr Neurol. 2016;23(2):143-50.

20. Kaal KJ, Aguiar M, Harrison M, McDonald PJ, Illes J. The clinical research landscape of pediatric drug-resistant epilepsy. J Child Neurol. 2020;35(11):763-6. https://doi.org/10.1177/0883073820 931255.

21. Eaton ML, Illes J. Commercializing cognitive neurotechnology: the ethical terrain. Nat Biotechnol. 2007;25(4):393-7. https://doi. org/10.1038/nbt0407-393.

22. Powell RA, Single HM. Focus groups. Int J Qual Health Care. 1996;8(5):499-504. https://doi.org/10.1093/intqhe/8.5.499.

23. Braun V, Clarke V. Using thematic analysis in psychology. Qual Res Psychol. 2006;3(2):77-101. https://doi.org/10.1191/14780 88706qp063oa.

24. Mcfadden D. Conditional logit analysis of qualitative choice behavior. In: Zarembka P, editor. Frontiers in economics. New York: Academic Press; 1974. p. 105-42.

25. Lancaster K. Consumer demand: a new approach. New York: Columbia University Press; 1972.

26. Harrison M, Rigby D, Vass C, Flynn T, Louviere J, Payne K. Risk as an attribute in discrete choice experiments: a systematic review of the literature. Patient. 2014;7(2):151-70.

27. Racine E, Dion M-J, Wijman CAC, Illes J, Lansberg MG. Profiles of neurological outcome prediction among intensivists. Neurocrit Care. 2009;11(3):345-52.

28. Wijnen BFM, de Kinderen RJA, Colon AJ, Dirksen CD, Essers BAB, Hiligsmann M, et al. Eliciting patients' preferences for epilepsy diagnostics: a discrete choice experiment. Epilepsy Behav. 2014;31:102-9.

29. Obadha M, Barasa E, Kazungu J, Abiiro GA, Chuma J. Attribute development and level selection for a discrete choice experiment to elicit the preferences of health care providers for capitation payment mechanism in Kenya. Health Econ Rev. 2019;9(1):30. https://doi.org/10.1186/s13561-019-0247-5. 


\section{Authors and Affiliations}

\section{Glory Apantaku ${ }^{1}$ Magda Aguiar ${ }^{1} \cdot$ K. Julia Kaal ${ }^{1}$ - Patrick J. McDonald ${ }^{2,4} \cdot$ Mary B. Connolly $^{3} \cdot$ Viorica Hrincu $^{2}$.} Judy llles ${ }^{2} \cdot$ Mark Harrison ${ }^{1,5}$

1 Faculty of Pharmaceutical Sciences, The University of British Columbia, Vancouver Campus, 4625-2405 Wesbrook Mall, Vancouver, BC V6T 1Z3, Canada

2 Division of Neurology, Department of Medicine, Neuroethics Canada, University of British Columbia, 2211 Wesbrook Mall, Koerner, S124, Vancouver, BC V6T 2B5, Canada

3 Division of Neurology, Department of Pediatrics, University of British Columbia, Vancouver, BC, Canada
4 Division of Neurosurgery, Department of Surgery, Faculty of Medicine, University of British Columbia, Vancouver, BC, Canada

5 Centre for Health Evaluation and Outcome Sciences, St. Paul's Hospital, Vancouver, BC, Canada 